\title{
Développement financier : cas des pays de la zone UEMOA (Burkina Faso, Côte d'Ivoire et Mali)
}

\section{SOMAN Kouadio Daouda ${ }^{1}$, NDOUNIAMA ONIONGUI Van-Brentano ${ }^{2}$ et HACHEMI Bentahar $^{3}$}

\author{
${ }^{1}$ Doctorant à l'Université Mohammed Premier, Oujda, Maroc \\ ${ }^{2}$ Doctorant à l'Université Mohammed Premier, Oujda, Maroc \\ ${ }^{3}$ Enseignant chercheur à l'Université Mohammed Premier, Oujda, Maroc
}

\begin{abstract}
Résumé : L'objet de ce papier est de connaître quel est le pays qui a le niveau de développement financier le plus élevé sur la période allant de 1975 à 2020. Pour se faire, nous avons utilisé l'analyse en composantes principales (ACP) afin d'avoir l'indice composite de chaque pays. Après comparaison des différents indices composites via l'ACP, les résultats montrent avec évidence que, pour le cas du Mali, la première valeur propre indique que la première composante principale explique plus de 71,509\% de l'inertie totale (et plus de 74,564\% respectivement 89,234\%) pour la Côte d'Ivoire et le Burkina Faso. Par-là, nous pouvons dire que le Burkina Faso a le niveau de développement financier le plus élevé.
\end{abstract}

Mots-clés : ACP, Développement financier, Indice composite, UEMOA.

\section{INTRODUCTION}

De nos jours, l'effet positif du développement financier sur la croissance économique anime plusieurs débats par contre les questions qui déterminent le niveau de développement financier restent partiellement discutées. En effet, l'analyse du rôle du développement financier peut remonter au moins à Bagehot (1873) qui prétend que l'étendue des marchés bien organisés en Angleterre peut améliorer l'allocation des ressources vers des investissements plus productifs. Par la suite, il y a eu les auteurs comme Schumpeter (1911), Hicks (1969) et Goldsmith (1969) qui ont également soutenu cette idée. Quant à Goldsmith (1969), il a trouvé un lien positif entre le développement financier et la croissance économique à partir d'une étude comparative avec des données de 35 pays sur la période allant de 1860 à 1963.

Aussi, courant ces dernières décennies, le cadre de pensée sur la répression financière proposé par McKinnon (1973) et Shaw(1973) a été la principale base intellectuelle de l'analyse des marchés financiers et conseils politiques. Réellement, il a été constaté qu'avant les années 1970, la plupart des pays en développement avaient été réprimés financièrement dans le sens où leurs systèmes financiers leur imposaient une fiscalité discriminatoire sous la forme de taux d'intérêt bas pour les dépôts, les réserves obligatoires élevées et la 
taxation des intermédiaires financiers. Selon McKinnon et Shaw, la répression financière réduit à la fois la quantité et la qualité des investissements dans l'économie en ce sens qu'un taux de dépôt inférieur à l'intérêt décourage les ménages de détenir des épargnes qui seraient utilisées pour financer les investissements productifs. Ainsi, les politiques répressives du gouvernement envers les systèmes financiers telles que les plafonds de taux d'intérêt, les réserves élevées requises, contrôle du crédit retardent le développement financier, et donc la croissance économique. A l'opposé, une libéralisation financière peut stimuler l'investissement et sa productivité et favoriser par la même occasion la croissance économique.

Toutefois, il semble que jusqu'en 1980, le secteur financier était toujours considéré comme répressif, fortement concentré dans les zones urbaines, averse au risque et n'offrant qu'une gamme limitée de services financiers en Afrique (Andrianaivo et Yartey, 2010). Cependant, pour intervertir la situation en prenant en considération l'effet positif du secteur financier notamment le secteur bancaire dans le progrès des activités économiques, plusieurs réformes ont vu le jour. Il était question de la restructuration des banques, du renforcement du cadre institutionnel, de la règlementation bancaire, de la promotion de l'autonomie de la politique monétaire et de la crédibilité des banques centrales (Ndikumana, 2003).

L'objectif de ces réformes était de renforcer la performance du secteur financier afin d'assurer intégralement son rôle d'allocation des ressources en faveur des investissements plus productifs. Ainsi, au sein de l'Union Economique et Monétaire Ouest-Africaine (UEMOA) ${ }^{1}$, ces réformes ont modifié la sphère financière dans le temps. En effet, le système financier s'est développé en comportant plus de banques, d'entreprises d'assurances et divers autres intermédiaires financiers dont le rôle dans la collette de l'épargne a augmenté. A ces entités, s'ajoutent les institutions de microfinance qui ont prospéré promptement malgré leurs différences (Ekomo et Avom, 2007).

Malheureusement, ces réformes n'ont pas pu atteindre les résultats escomptés car la structure du système financier demeure presque inchangée. Le système financier des pays d'Afrique de la zone UEMOA s'appuie beaucoup plus sur les intermédiaires que sur les marchés financiers développés. Il semble même que ces derniers sont quasi-inexistants dans la zone. Cela sous-entend que le financement des activités économiques dans ses pays est principalement basé sur les ressources provenant des banques que sur celles provenant des marchés financiers. Tout en sachant que tous les pays de l'UEMOA sont soumis aux mêmes décisions et stratégies élaborées par la Banque Centrale des Etats de l'Afrique de l'Ouest (BCEAO), nous nous sommes posés la question à savoir, lequel des trois pays choisis détient le développement financier le plus élevé ?

L'apport de ce papier consiste à travailler exclusivement sur le niveau de développement financier des différents pays de la zone UEMOA en l'occurrence Burkina, Côte d'Ivoire et Mali puisque la plupart des

${ }^{1}$ UEMOA regroupe 8 pays à savoir le Bénin, le Burkina Faso, La Côte d'Ivoire, la Guinée-Bissau, le Mali, le Niger, le Sénégal et le Togo. 
travaux qui ont été abordés dans ce champ, ont mis l'accent profond sur l'impact du développement financier sur la croissance économique ${ }^{2}$.

Cette étude sera structurée de la manière suivante : la section 2 consistera à définir avec précision les différents concepts, faire un état des lieux et aussi le choix des variables. Quant à la troisième section, elle sera dédiée à la construction de l'indice composite. Et enfin, la dernière section sera consacrée à la conclusion.

\section{DEFINITIONS DES CONCEPTS, ETATS DES LIEUX ET CHOIX DES VARIABLES}

\section{A. Définitions}

Un système financier est un ensemble d'institutions, notamment les banques, les compagnies d'assurance et les bourses, qui permettent l'échange de fonds. En effet, les emprunteurs, les prêteurs et les investisseurs échangent des fonds courants pour financer des projets, qu'ils soient destinés à la consommation ou à des investissements productifs, et pour rechercher un rendement sur leurs actifs financiers. Le système financier est destiné à mettre en relation l'offre et la demande de capitaux. Il implique également un ensemble de règles et de pratiques que les emprunteurs et les prêteurs utilisent pour décider quels projets sont financés, qui finance les projets et les conditions des accords financiers.

Selon Levine (2005), le développement financier est le processus par lequel les instruments, les marchés et les intermédiaires financiers améliorent le traitement de l'information, la mise en œuvre des contrats et la réalisation des transactions. Il permet ainsi au système financier de mieux exercer les fonctions ci-après :

- la facilitation des échanges de biens et de services ;

- la mobilisation et la collecte de l'épargne ;

- la production d'information sur les investissements envisageables et l'allocation de l'épargne ;

- la répartition, la diversification et la gestion du risque ;

- le suivi des investissements en exécution et le contrôle de la gouvernance.

Pour Rafael Cezar $(2012)^{3}$, le développement financier surgit lorsque les institutions financières réduisent les frictions existantes et facilitent l'allocation des ressources. Il doit exprimer l'efficacité du système dans l'exécution de chacune des six principales fonctions financières à savoir

- la mobilisation du capital ;

- la production et la diffusion d'informations sur les agents économiques ;

- la maîtrise et le partage du risque ;

2 (GELBARD E., GULDE A., MAINO R., 2014).

${ }^{3}$ (CEZAR R., 2012). 
- le suivi des investissements (de façon à ce que le capital soit utilisé de façon optimale) et la gouvernance des entreprises ;

- la réduction des coûts de transactions ;

- la liquidité des placements financiers en procurant ainsi une allocation optimale des ressources.

Nous pouvons retenir qu'on parle développement financier lorsque le système financier réalise avec aisance les fonctions citées par Levine (2005) et Rafael (2012).

\section{B. Etat des lieux}

Le système financier de l'Union Economique et Monétaire Ouest- Africaine (UEMOA) est dominé par les établissements bancaires. On y trouve une seule bourse à savoir la Bourse Régionale des Valeurs Mobilières (BRVM) qui a son siège à Abidjan (Côte d'Ivoire) et qui est commune aux pays de cette Union. En effet, le financement de l'économie provenant des bourses est très faible comparé à celui émanant des banques. Nous allons faire un inventaire dans chaque pays.

\section{- $\quad$ La Côte d'Ivoire}

La Côte d'Ivoire est un pays de l'Afrique de l'ouest qui a connu un boom économique grâce aux cours du café et du cacao qui s'est accompagné d'une croissance économique soutenue ${ }^{4}$ jusqu'en 1978. Cependant, après cette époque, l'économie ivoirienne a été frappée par une crise au cours de laquelle le secteur financier a graduellement ressenti les conséquences de la baisse du niveau de l'activité économique. Ainsi la relance économique a débuté par un Programme d'Ajustement du Secteur Financier (PASFI) initié par la Banque Mondiale dont l'objet était de promouvoir dans un cadre règlementaire et institutionnel approprié, un système financier solvable, diversifié et élargi, capable de couvrir les besoins d'épargne et de crédit des agents économiques, de financer le développement sur les bases saines sans porter atteinte à l'intégrité des fonds confiés par le système. Ainsi, la mise en place des mesures a permis d'avoir des résultats satisfaisants avec la restructuration de toutes les banques et la liquidation de celles qui étaient compromises.

Malgré cette restructuration entamée depuis fort longtemps, l'OCDE (2016) affirma que le système financier est faiblement développé et son soutien à l'économie réelle est largement insuffisant en Côte d'Ivoire. Pour elle, le niveau de développement du secteur financier dans le pays est inférieur à celui de plusieurs pays africains et ne permet pas de soutenir pleinement l'économie réelle. En effet, l'octroi de crédits bancaires au secteur privé est insuffisant soit $20 \%$ du PIB. D'après la banque mondiale, l'accès limité au financement crée une contrainte majeure pour de nombreuses entreprises. Le système financier ivoirien comme pour la plupart des pays de l'UEMOA, est peu diversifié et les options de financement alternatif sont faibles. Pour terminer, nous pouvons ajouter qu'une grande partie de la population ivoirienne est localisée dans une zone hors des itinéraires de financement formel ce qui fait qu'environ $70 \%{ }^{5}$ des ivoiriens ne détiennent pas de compte bancaire.

${ }^{4}$ (COGNEAU D. et MESPLE-SOMPS S. 2002).

${ }^{5}$ BCEAO(2019), «Rapport sur la situation de l'inclusion financière dans l'UEMOA au cours de l'année 2018», www.bceao.int/sites/default/files/2019-10/Rapport_Annuel_2018_Situation_Inclusion_Financiere.pdf. 
Le système financier ivoirien reflète donc des inégalités territoriales et un clivage urbain-rural (la moitié des institutions est à Abidjan).

En 2018, la Direction Générale du Trésor et de la Comptabilité a initié un Réseau des Acteurs de $1^{\prime}$ Education Financière ${ }^{6}$ afin de renforcer l'éducation financière des populations notamment celles vivant en milieu rural. La population rurale ignore non seulement l'importance de détenir un compte bancaire mais aussi elle n'a pas confiance ${ }^{7}$ à une institution qui pourrait gérer ses avoirs financiers. Toutefois, pour changer cette perception aux yeux de cette population, des campagnes de sensibilisation, des ateliers de cadrage et de validation de la Stratégie Nationale d'Inclusion Financière ont été initiés par l'Agence de Promotion de l'Inclusion Financière dans plusieurs localités, dont l'objet est de les inciter à avoir un compte bancaire.

\section{- Burkina Faso}

Le Burkina est un pays du Sahel disposant d'une économie basée essentiellement sur l'agriculture même si les exportations aurifères ont progressé. En effet, le Burkina comme tout autre pays, a besoin des ressources financières pour développer ses activités économiques tandis que son système financier est principalement dominé par le secteur bancaire qui malgré des récents progrès, les difficultés d'accès aux services financiers persistent. Selon le rapport sur la situation financière de l'inclusion financière dans l'UEMOA au cours de l'année 2018, seulement $21,3 \%{ }^{8}$ de la population Burkinabé détient un compte bancaire au sens strict et sens large ${ }^{9}$, seulement $41,3 \%$.

Ces faibles intérêts pour la bancarisation peuvent être expliqués en partie par l'existence de barrières structurelles telles que les faibles revenus, le coût des prestations, l'éloignement du point de service, des exigences documentaires complexes. En plus, il se trouve qu'il y a des différences croissantes dans l'accès au financement en fonction de l'éducation, des revenus et aussi du fait qu'en général, les femmes sont moins susceptibles que les hommes d'avoir un compte bancaire. Par conséquent, l'utilisation d'arrangements informels tels que l'emprunt auprès de la famille et des amis reste prédominante.

Pour booster le secteur financier, les autorités régionales et nationales ont lancé des mesures pour améliorer l'inclusion financière, dont les plus récentes sont :

- Au niveau régional, l'adoption, par le Conseil des Ministres de l'UEMOA ${ }^{10}$, d'une stratégie d'inclusion financière pour la période 2016-2020, fondée sur cinq piliers, dont la promotion de cadres réglementaires et juridiques efficaces et le renforcement du secteur de la microfinance et littéraire financière;

- L'élaboration d'une stratégie nationale d'inclusion financière, avec l'aide du Fonds d'équipement des Nations Unies (UNCDF).

\footnotetext{
${ }^{6}$ Idem.

${ }^{7}$ Cette dernière pense que si la banque fait faillite, elle va tout perdre, le mieux serait de garder l'argent chez elle.

${ }^{8}$ BCEAO(2019), «Rapport sur la situation de l'inclusion financière dans l'UEMOA au cours de l'année 2018», www.bceao.int/sites/default/files/2019-10/Rapport_Annuel_2018_Situation_Inclusion_Financiere.pdf.

${ }^{9}$ Le taux de bancarisation strict mesure le pourcentage de la population adulte détenant un compte dans les banques, les services postaux, les caisses nationales d'épargne et le Trésor tandis que celui de bancarisation élargi évalue le pourcentage de la population adulte titulaire de comptes dans les banques, les services postaux, les caisses nationales d'épargne et le Trésor, auquel s'ajoute celui des détenteurs de comptes dans les institutions de microfinance.

${ }^{10} \mathrm{BCEAO}(2019)$, «stratégie régionale d'inclusion financière dans 1'UEMOA : Vision, Objectif, Axes stratégiques et Dispositif institutionnel de mise en œuvre», Direction Générale de la Stabilité et de l'Inclusion Financières, Dakar.
} 
Compte tenu des faibles revenus de la majorité de la population, les recommandations formulées par les institutions de développement incluent également la création d'un cadre de réduction ou de suppression des frais de tenue de compte afin d'approfondir la couverture bancaire. Les activités de microfinance, notamment la collecte d'épargne et l'octroi de crédit, sont soumises à autorisation préalable du Ministère de l'Economie et des Finances. La supervision est assurée par la Direction Générale du Trésor et de la Comptabilité Publique (DGTCP) à travers la Direction de la Microfinance.

- Mali

Le Mali est un vaste pays enclavé d'Afrique de l'ouest disposant d'une économie en grande partie rurale avec plus des deux tiers de la population vivant de l'agriculture particulièrement le coton. En effet, l'économie malienne est fortement dépendante de l'extérieure et très exposée aux aléas climatiques en dépit de multiples défis auxquels le pays fait face. L'un de ces défis est le faible niveau de son développement financier, caractérisé par le secteur bancaire. Ce dernier a un niveau de développement relativement faible et peu diversifié avec une disponibilité limitée des actifs financiers.

Le secteur bancaire malien était composé des banques publiques durant les premières années de son indépendance. C'est à partir de $1989^{11}$ que le peuple malien a amorcé la restructuration du système bancaire et financier par la mise en place des mécanismes plus flexibles de régularisation monétaire. Il s'est accompagné de l'abandon de la politique sélective du crédit associant le relâchement des contraintes administratives à un renforcement de la surveillance bancaire, dans le cadre d'une libéralisation graduelle du marché du crédit. A cela, s'est ajoutée l'adoption des dispositions pour assurer une mobilisation accrue de l'épargne intérieure, une diversification et un approfondissement financier en vue d'une meilleure allocation des ressources au financement du secteur productif et de l'investissement.

Malgré ces réformes entamées depuis 1989, le taux de bancarisation demeure très faible car d'après le rapport sur la situation financière de l'inclusion financière dans l'UEMOA au cours de l'année 2018, seulement $23,3 \%$ de la population malienne détient un compte bancaire au sens strict et $36,7 \%$ au sens large. Ce faible taux peut se justifier par le fait qu'en dépit de la présence des banques, le modèle coopératif ou associatif prédomine l'ensemble des approches adoptées par la finance inclusive ${ }^{12}$. Au Mali, il existe beaucoup de coopératives financières qui contribuent nécessairement au développement social et à l'amélioration des conditions de vie des communautés. Ce modèle coopératif privilégie la propriété collective en accentuant la quête d'impact de ce financement sur la réduction de la pauvreté individuelle et collective.

\footnotetext{
${ }^{11}$ (N'DIAVE M., 2004).

${ }^{12}$ https://www.international.gc.ca/world-monde/stories-histoires/2019/mali-finance.aspx?lang=fra.

L'inclusion financière est un processus qui permet aux individus et aux entreprises d'accéder aux services financiers de base (dépôts et transferts de fonds, paiements, épargne, crédit, assurance) fournis par les institutions financières formelles (Banque de France, 2014).
} 
- Une approche de synthèse

$\mathrm{Au}$ vu de ce qui précède, nous pouvons dire que les pays membres de l'UEMOA n'ont pas le même niveau de développement financier par contre ils ont tous débuté des réformes de restauration à la fin des années 1980. On retient également que le système financier de l'Union est dominé par le secteur bancaire et que malgré la mise en œuvre de plusieurs reformes, ce secteur reste peu développé. Et ce, le taux de bancarisation, que ce soit strict ou élargi demeure très faible. Les services bancaires sont moins désirés en Afrique, les populations rurales ne maitrisent pas les avantages de détenir un compte bancaire et donc, n'opèrent que peu d'activités avec les banques et pourtant les banques ne peuvent survivre sans les clients.

Voici un tableau qui présente les taux de bancarisation dans les trois pays de notre travail.

Tableau 1 : Taux de bancarisation strict et élargi en \% en 2018.

\begin{tabular}{|l|c|c|}
\hline Pays & $\begin{array}{l}\text { Taux de bancarisation strict en } \\
\mathbf{2 0 1 8}\end{array}$ & Taux de bancarisation élargi en 2018 \\
\hline Burkina & 21,3 & 41,3 \\
\hline Côte d'Ivoire & 21,6 & 32,6 \\
\hline Mali & 23,3 & 36,7 \\
\hline
\end{tabular}

Source: élaboration des auteurs sur la base des données du Rapport sur la situation de l'inclusion financière dans l'UEMOA au cours de l'année 2018.

Il parait clair qu'au sens strict, le Mali détient le taux de bancarisation le plus élevé par contre au sens large, c'est le Burkina Faso qui dispose le taux de bancarisation le plus élevé suivi par le Mali. Toutefois, au sein de l'Union, c'est le Togo qui détient le taux de bancarisation le plus élevé que ce soit au sens large comme au sens strict (26,8\% au sens strict et $85,4 \%$ au sens large). Parmi les 8 pays membres, c'est le Niger qui détient le taux de bancarisation le plus faible au sens strict (soit $5,8 \%$ ) et au sens large, c'est la Guinée Bissau qui se classe en dernière position avec un taux de $11,8 \%$.

Il est nécessaire de dire que l'utilisation de mobile money a contribué, de manière significative, à l'amélioration du taux d'inclusion financière des populations dans l'Union. De plus en plus, les populations s'intéressent au mobile money au détriment des services bancaires. En 2017, près de 16943 milliards de FCFA soit équivalent $30,68^{13}$ milliard de dollars américain ont été transité par le mobile money contre 11501 milliards de FCFA soit 20,82 milliard de dollars américain en 2016. En effet, la Côte d'Ivoire totalise elle seule 37,9\% des comptes de monnaie électronique en 2016 suivi de Burkina Faso (13,8\%) et le Mali (13,5\%). En 2018 ${ }^{14}$, l'activité financière est restée dominée par la Côte d'Ivoire avec près 23,6 millions de comptes de monnaie électronique de l'Union (soit 37,4\%), suivie du Burkina Faso, 9,6 millions de comptes.

Le paiement électronique via la téléphonie mobile a pris de l'ampleur dans l'Union depuis la propagation de la pandémie liée au Covid-19. En vérité, la BCEAO a décidé, en collaboration avec la communauté des émetteurs de monnaie électronique de l'Union, d'assouplir provisoirement les

131 dollars $=552,3069$ FCFA.

${ }^{14}$ https://www.sikafinance.com/marches/uemoa-23-533-milliards-fcfa-echanges-via-le-mobile-money-en-2018 19841. 
diverses mesures de la réglementation relative à la monnaie électronique afin de limiter la propagation du dit virus.

Pour terminer, l'on peut affirmer que l'utilisation de la téléphonie mobile par le secteur financier a contribué de manière significative au relèvement du taux global d'utilisation des services financiers dans l'UEMOA. Le mobile money a facilité les transactions de crédits des populations quel que soit leur situation géographique dans la zone. Les populations n'ont plus rien à craindre en ce que qui concerne les transactions tant qu'elles se trouvent dans une localité couverte par un réseau mobile. Elles réceptionnent et font des transactions en temps et en heure.

\section{Choix des indicateurs}

La formation de notre indice se base sur les variables les plus utilisées dans la littérature financière pour les pays en développement. Ces indicateurs sont quantitatifs et bancaires du fait que le secteur financier de l'Union (des pays étudiés) repose principalement sur l'intermédiation bancaire. Nous avons mentionné cihaut que la zone UEMOA dispose d'une seule bourse dont son impact dans le financement des entreprises est quasiment nul. La contrainte supplémentaire également prise en compte est la disponibilité des données pour la période étudiée.

-Masse monétaire en pourcentage du PIB : Cet indicateur prend en compte tous les moyens de paiements disponibles dans l'économie. Nous avons utilisé la masse monétaire au sens large à cause de l'indisponibilité des données mais également nous pensons que cette masse monétaire au sens large est égale à M2 (en \% PIB) vu que les titres négociables qui s'ajoutent à M2 pour donner M3 sont quasi-inexistants dans l'Union. La masse monétaire M2 est l'indicateur le plus utilisé dans la littérature (King et Levine, 1993; World Bank, 1989 McKinnon (1973)).

-Crédit intérieur fourni au secteur privé en pourcentage du PIB : Il comprend tous les crédits accordés aux différents secteurs privés à l'exception du crédit au gouvernement central, qui est net. Selon Levine c'est le meilleur indicateur pour mesurer le développement financier car pour lui, le système financier susceptible de collecter les dépôts et les transférer vers le secteur privé sous forme de crédit, est capable d'assurer les autres fonctions économiques du système financier déjà présenté dans la partie théorique.

-Crédit intérieur fourni au secteur privé par les banques en pourcentage du PIB : Cet dernier indicateur permet de mesurer le rôle des banques dans le financement des activités économiques donc l'allocation des ressources financières (une possibilité que la première variable n'offre pas). Le crédit accordé au secteur privé en pourcentage du PIB est lié à la quantité ainsi qu'à la qualité de l'investissement.

\section{INDICE COMPOSITE}

Concernant la construction de l'indice composite, nous allons utiliser la méthode de l'analyse en composantes principales $(\mathrm{ACP})$ via le logiciel SPSS. Il est très approprié ou adéquat à notre sens de définir l'indice composite. L'indice composite est un indice synthétique c'est-à-dire il regorge en son sein les trois (3) variables considérées en l'occurrence le ratio de liquidité (masse monétaire); le crédit intérieur fourni au secteur privé et enfin le crédit intérieur fourni au secteur privé par les banques.

Il faut souligner que l'analyse en composantes principales est une méthode factorielle qui a pour objet de représenter les individus et les variables quantitatives sous forme d'un tableau dans un espace réduit. Cela 
revient à déterminer les axes (factoriels) qui passent par le maximum de points ${ }^{15}$. Il faut aussi noter que l'ACP est constituée de plusieurs axes factoriels mais nous allons juste nous intéresser à l'information apportée par la première composante principale c'est à dire l'indicateur composite, plus l'information ou bien la variance est grande plus la confiance en notre indice l'est aussi. Toutefois, trois (3) conditions sont essentielles pour réaliser l'ACP :

- premièrement, l'indice de $\mathrm{KMO}^{16}$;

- deuxièmement, la $\mathrm{p}$-value du test de Bartlett doit-être significative avec un $(\mathrm{p} \approx 0)$;

- troisièmement, les variables doivent-être fortement corrélées entre elles.

Dans le cadre de notre travail, l'indice de KMO qui vérifie la pertinence de notre analyse du point de vu statistique de chacun de nos pays est supérieur à $5 \%$, le test de Bartlett est significatif, avec un $(\mathrm{p} \approx 0)$ pour les trois (3) pays de l'échantillon. Et enfin, nos variables sont fortement corrélées entre elles. Par conséquent, nous pouvons appliquer l'analyse en composantes principales dans le cadre de notre étude.

Les résultats obtenus par la méthode d'ACP se trouvent dans le tableau ci-dessous :

Tableau 2: Les résultat de l'ACP

\begin{tabular}{|c|c|c|c|c|c|c|c|c|}
\hline \multirow[t]{2}{*}{ Pays } & \multicolumn{2}{|c|}{1 ère val propre } & \multicolumn{3}{|c|}{2 ères val propre } & \multicolumn{3}{|c|}{3 ères val propre } \\
\hline & Vce & $\begin{array}{l}\text { Variabilité } \\
(\%)\end{array}$ & Vce & $\begin{array}{l}\text { Variabilité } \\
(\%)\end{array}$ & $\begin{array}{l}\% \\
\text { cumulé }\end{array}$ & Vce & $\begin{array}{l}\text { Variabilité } \\
(\%)\end{array}$ & $\begin{array}{l}\% \\
\text { cumulé }\end{array}$ \\
\hline Côte d'Ivoire & 2,237 & 74,564 & 0,763 & 25,428 & 99,992 & 0,000 & 0,008 & 100 \\
\hline Mali & 2,145 & 71,509 & 0,304 & 10,118 & 99,352 & 0,19 & 0,648 & 100 \\
\hline Burkina Faso & 2,677 & 89,234 & 0,304 & 10,118 & 99,352 & 0,19 & 0,648 & 100 \\
\hline
\end{tabular}

Source: élaboré par les auteurs à partir des données de la banque mondiale sous SPSS

Pour le cas de la Côte d'ivoire, la première valeur propre indique que la première composante principale explique plus de 74,564\% de l'inertie totale, pour le Mali, elle explique plus de 71,509\% et enfin concernant le Burkina Faso, elle est de 89,234\%. Au travers de ces résultats, nous apercevons que c'est le Burkina Faso qui détient la valeur propre la plus élevée.

Figure 1 L'évolution de l'indice de développement financier de 1975 à 2020.

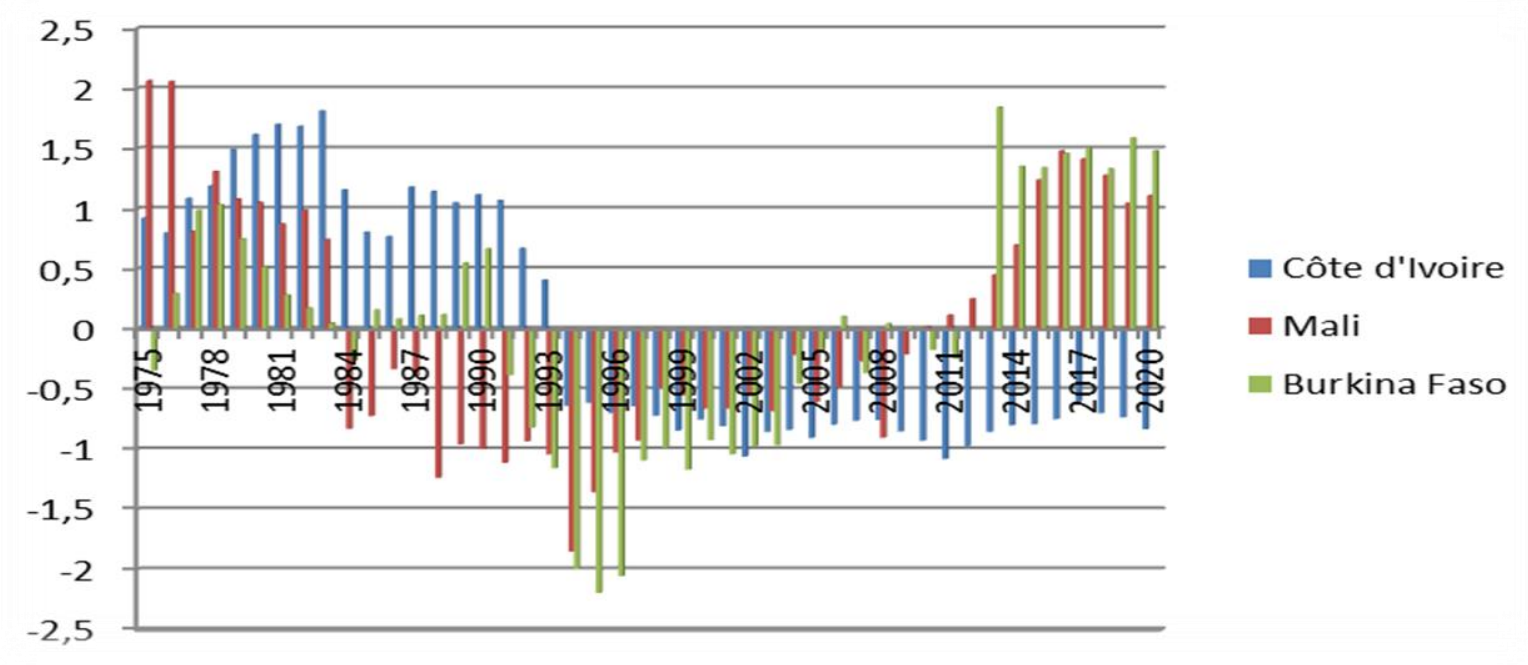

15 (KASMI Amel, 2013)

${ }^{16}$ La mesure de Kaiser-Meyer-Olkin est un indice d'adéquation de la solution factorielle et une mesure de la qualité de l'échantillonnage. Un KMO élevé (proche de 1) indique que l'analyse factorielle est une méthode appropriée et pertinente pour analyser les données. En revanche, un KMO inférieur à 0,5, signifie que les variables ne partagent pas assez de variance pour que l'analyse factorielle soit adéquate. 
L'indice composite de développement financier que nous avons construit est un indice synthétique obtenu via la méthode ACP. Son évolution dépend aussi bien du temps qu'à sa valeur. Nous remarquons qu'entre la période de 1975-1977, le Mali détenait le niveau de développement financier le plus élevé par rapport à ses voisins. Cependant, nous observons une chute considérable de l'indice de ce dernier à partir de 1984. Cela s'explique par un niveau de développement relativement faible et peu diversifié avec une disponibilité limitée des actifs financiers. De 1984 à 1994, sa situation s'est aggravée jusqu'à 2009 pour les mêmes anomalies évoquées ci-dessus. Ce n'est qu'à partir de 2010 que son niveau de développement financier a commencé à s'améliorer jusqu'en 2020 grâce à la restructuration du système bancaire et financier par la mise en place des mécanismes plus flexibles de régulation monétaire. De 1975 à 1993, nous constatons que le niveau de développement financier de la Côte d'Ivoire n'était pas mal vu qu'en 1983 parmi les trois (3) pays de l'étude, elle occupait la première place. Cet exploit émane de la restructuration de son système bancaire initiée par ce pays à l'aide des institutions de Brettons Woods au début des années 80. Néanmoins, de 1994 jusqu'à 2020, nous constatons un faible niveau de développement. Ce résultat reflète les crises qu'a connues ce pays. C'est un effet paradoxal dans la mesure où en Côte d'Ivoire, la libéralisation financière a été amorcée en 1989 avec la nouvelle politique de la monnaie et du crédit de la BCEAO. Toutefois, en Côte d'Ivoire nous avons observé une dégradation du développement financier. Enfin le Burkina Faso, de 1975 à 1990, son niveau de développement financier était le plus faible des pays de l'étude. Cela peut être vu dans le contexte du coup d'Etat qui a entrainé la mort de l'Ancien Président Burkinabè Thomas Sankara le 15 octobre 1987. L'année 1995 est marquée par un désastre absolu de ce pays notamment la mauvaise conjoncture qu'il avait connu. A partir de 2014 jusqu'à la fin de la période, ce dernier devient le pays qui a fait d'énormes améliorations par rapport aux deux (2) autres, cela est le fruit d'un développement financier qui résulte des normes mentionnées ci-haut (voir état des lieux du Burkina Faso).

\section{CONCLUSION}

Pour conclure, ce travail avait pour mission de déterminer le pays qui connait un développement financier le plus élevé parmi nos trois pays de l'UEMOA en l'occurrence la Côte d'Ivoire, le Mali et la Burkina Faso sur la période allant de 1975 à 2020. Il s'avère que c'est le Burkina Faso qui détient le niveau de développement le plus élevé sur la période considérée. Nous pouvons vérifier à travers son indice composite (ses vecteurs propres de F1 sont supérieures aux deux (2) autres F1 des deux autres pays en termes de variance et de variabilité). Pour y parvenir, nous avons fait l'ACP dans le but d'avoir notre indice composite au sein de chaque pays tout en se basant sur le F1 c'est-à-dire la composante qui contient plus d'informations. En gros, nos pays de l'étude ont un mauvais niveau de développement ce qui nous a permis de considérer juste la masse monétaire M2 vu qu'il n'existe presque pas la masse monétaire M3 plus spécifiquement le titre de créance. Ce travail recommande que tous ces pays doivent mettre en place des politiques adéquates afin de s'approcher de leur pays voisins comme le Maghreb (Algérie, Maroc et Tunisie).

\section{BIBLIOGRAPHIE}

[1] M. Andrianaivo, et C. Yartey, "Understanding the Growth of African Financial Markets", African Development Review, 2010, pp. $394-418$.

[2] W. Bagehot, “A Description of the Money Market”, EP Dutton and Company Reprint 1920 New York, 1873.

[3] L. Bamba, "Structure et efficacité du Secteur Financier Ivoirien", Sortir du Sous-Développement: Quelles Nouvelles pistes pour l'Afrique de l'Ouest, T. 2: Financement et Coopération, L'Harmattan, 2011, pp.255-278.

[4] M. Barry, "Développement financier et croissance économique: études théoriques et applications sur l'UEMOA et la CEDEAO”, Economies et finances, Université de Franche-Comté, Français, 2012. 
[5] M. Batuo, F. Guidi, et K. Mlambo, "Financial Development and Income Inequality: Evidence from African Countries", MPRA Paper, University Library of Munich, Germany, 2010.

[6] Bceao, "Rapport sur la situation de l'inclusion financière dans l'UEMOA au cours de l'année 2018”, 2019.

[7] -Bceao, "Stratégie régionale d'inclusion financière dans l'UEMOA : Vision, Objectif, Axes stratégiques et Dispositif institutionnel de mise en œuvre", Direction Générale de la Stabilité et de l'Inclusion Financières, Dakar, 2019.

[8] S Beji, "Le développement financier pour les pays du sud de la Méditerranée à l'épreuve de la mondialisation financière", Economies et finances. Université Paris-Nord - Paris XIII, 2009.

[9] R. Cezar, "Un nouvel indice du développement financier", Université Paris Dauphine, 2012.

[10] Cogneau, et S. Mesple-somps, "L'économie ivoirienne, la fin du mirage ? ”, Document de travail DIAL / Unité de Recherche CIPRÉ, étude réalisée dans le cadre du programme "Afrique émergente" du Centre de Développement de l'OCDE débutée en 1997, 2002.

[11] Cogneau, et S. Mesple-somps, "La Côte d'Ivoire peut-elle devenir un pays émergent ? ", Programme d'étude "Afrique émergente" Centre de Développement, OCDE, Paris, 1999.

[12] S. Coulibaly, "L'inclusion Financière à Travers la Monnaie Mobile : Analyse de la Décision sur L'utilisation des Comptes de Monnaie Mobile dans les Pays de l'UEMOA", Document de politique générale, Consortium pour la Recherche Économique en Afrique, African Economic Research Consortium, 2020.

[13] S. Ekomo, et D. Avom, “Quinze ans de restructuration bancaire dans la CEMAC : qu'avons-nous appris ?", Economie financière, 2007, pp. 183-205.

[14] L. Esso, "Développement financier, croissance économique et inégalités de revenus entre les pays de l'UEMOA", Consortium pour la recherche économique et sociale, Université Cheikh Anta Diop de Dakar, Faculté des Sciences Juridiques Economiques et de Gestion, Laboratoire d'analyse des politiques publiques, 2009.

[15] Gelbard, A. Gulde, et R. Maino, "Développement financier en Afrique Subsaharienne : les enjeux pour une croissance soutenue", Revue d'économie financière, 2014/4 N 116 | pages 19 à 42.

[16] R. Goldsmith, "Financial Structure and Development”, New Haven, CT: Yale, 1969.

[17] S. Guillaumont, et K. Kpodar, "Développement financier, instabilité financière et réduction de la pauvreté ", CNRSUniversité d'Auvergne, 2004.

[18] J. Hicks, “A Theory of Economic History, Oxford”, Claredon Press, 1969.

[19] Kasmi, "Etude des niveaux de développement financier des trois pays du Maghreb (Algérie, Maroc et Tunisie) ", Ecole nationale polytechnique d'Alger, vol.2, 2014, pp.146-148.

[20] R. Levine, "Finance and growth: theory and evidence", in P. Aghion and S. Durlauf (eds.), Handbook of Economie Growth, Amsterdam: North-Rolland, 2005, pp. 866-934.

[21] R. Mckinnon, "Money and Capital in Economic Development”, Washington, DC: Brookings Institution, 1973.

[22] E. Meskinis, et M. Zouheirm, "Le développement financier et la croissance économique : Cas des pays de la région MENA (Moyen orient Nord-africain)", Revue Internationale des Sciences de Gestion "Volume 3: Numéro 3", 2020, pp:425-442.

[23] E. Nazima, A. Kausar, M. Awais, H. Affandi, R. Saghir, et S. Qaim, "Investigating the Institutional Determinants of Financial Development: Empirical Evidence from SAARC Countries”, SAGE Journals, 2021.

[24] L. Ndeffo, O. Edokat, et C. Toukam, "Développement financier et croissance économique dans les pays africains de la zone franc : le rôle de la gouvernance”, European Scientific Journal February 2015, edition vol.11, No.4.

[25] L Ndikumana, "Financial markets and economic development in Africa", dans Emmanuel Nnadozie, African Economic Development, Academic Press, 2003, pp. 373-403.

[26] M. N'diave, “Approfondissement financier et croissance économique au Mali”, mémoire de fin d'étude soutenu pour l'obtention du Diplôme D'Etudes Approfondies en Economie, 2004, en ligne.

[27] M. Odhiambo, S. Nyasha, M Zerihun, et C. Tipoy, "Financial Development in Africa", Working Paper 16/2018 October 2018.

[28] J. SCHUMPETER, “The Theory of Economic Development”, Cambridge, MA: Harvard University Press, 1911.

[29] E. Shaw, "Financial Deepening in Economic Development", Oxford University Press, 1973.

[30] N. Tanimoune, "Les déterminants de la profitabilité des banques dans l’UEMOA : une analyse sur les données de panel”, Notes d'information et Statistiques - N 539 - Août/Septembre 2003 - Etudes et Recherche. 